\title{
NOTES
}

\section{Heat-treatment and Displacement of Adsorbed Lysozyme Layers}

\author{
R. J. MARsh,,${ }^{1, \dagger}$ R. A. L. Jones, ${ }^{2}$ and M. SfERrazZA ${ }^{3, \dagger}$ \\ ${ }^{1}$ Cavendish Laboratory, University of Cambridge, Madingley Road, Cambridge, UK \\ ${ }^{2}$ Department of Physics and Astronomy, University of Sheffield, Sheffield, UK \\ ${ }^{3}$ Département de Physique, Université Libre de Bruxelles, \\ Boulevard du Triomphe, CP 223, B-1050 Bruxelles, Belgique
}

(Received June 1, 2005; Accepted June 20, 2005; Published October 15, 2005)

KEY WORDS Globular Protein / Lysozyme / Ellipsometry /

[DOI 10.1295/polymj.37.789]

The adsorption of globular proteins at solid/liquid interfaces is an active area of research. ${ }^{1-3}$ In particular, efforts have been directed to the investigation of conformational changes undergone by the protein molecules upon adsorption using a variety of techniques including ellipsometry, ${ }^{4,5}$ neutron reflection, ${ }^{6-8}$ circular dichroism, ${ }^{2}$ Fourier Transform InfraRed Spectroscopy in the Attenuated Total Reflection mode (FT IR-ATR), ${ }^{9-12}$ and differential scanning calorimetry. ${ }^{13,14}$ Also of considerable interest are any structural changes undergone by a pre-adsorbed protein layer under external factors, as the heating and the displacement by small molecules.

In our previous work using ellipsometry, FT IR and neutron reflection, we have studied the adsorption and displacement by surfactant of the globular protein beta-lactoglobulin on hydrophilic and hydrophobic surfaces. ${ }^{4,6}$ This brief communication reports an investigation on the heat-treatment of adsorbed layers of lysozyme on hydrophobic and hydrophilic surfaces.

Lysozyme is an enzyme with lytic and bactericidal activity. It is involved in the hydrolysis of beta(1-4) glycosidic linkages of carbohydrates in cell walls. ${ }^{15}$ Lysozyme has a net positive charge at neutral $\mathrm{pH}$. The relevant properties of lysozyme are the following: the molecular weight is $14100 \mathrm{~g} \mathrm{~mol}^{-1}$; the isoelectric point 11; the charge per molecule at $\mathrm{pH}$ of 7 is $+7 \mathrm{e}$, the shape of the protein is ellipsoidal with diameter $30 \AA$ and length $45 \AA$; the partial specific volume is $0.712 \mathrm{~mL} \mathrm{~g}^{-1}$ and the refractive index increment $d n / d c$ at $25^{\circ} \mathrm{C}$ and $\mathrm{pH} 7$ is $0.1856 \mathrm{~mL} / \mathrm{g}$. The protein was obtained from Sigma Chemical Co., and had been 3 times crystallised and lyophilised, then freeze-dried (L-6876: Lot No. 65F-8170). The non-ionic surfactant used for the displacement studies was octaethylene glycol mondodecyl ether $\left(\mathrm{C}_{12} \mathrm{E}_{8}\right)$. The molecular weight of the surfactant is $538.77 \mathrm{Da}$, and the critical micelle concentration is $0.092 \mathrm{mmol} \mathrm{dm}^{-3} .{ }^{16}$ The surfactant was obtained from Nikko Chemical Co., Tokyo, Japan.

Hydrophobic surfaces were obtained by grafting octadecyltrichorosilane (OTS) of molecular weight of $387.7 \mathrm{Da}$ onto hydrophilic silicon surfaces. The grafting procedure has been described previously., The OTS layer was characterised by the contact angle technique, and ellipsometry. The contact angles were in the range of $105 \pm 5$ degrees. The ellipsometry gave a thickness for the OTS layer of $27 \pm 6 \AA$. This is equal to a surface excess of octadecane of $1.6 \pm$ $0.5 \mathrm{mg} \mathrm{m}^{-2}$, taking the molecular weight of the hydrophobic layer of $254.5 \mathrm{Da}$, density of $0.7768 \mathrm{~g} \mathrm{~m}^{-2}$, and a refractive index of 1.439 at $589 \mathrm{~nm} .{ }^{17}$ The effective refractive index increment of octadecane was determined to be $0.1367 \mathrm{~mL} \mathrm{~g}^{-1}$. The value of the surface excess corresponds to a molar excess of $6.4 \pm 1.9$ $\mu \mathrm{mol} \mathrm{m}{ }^{-2}$, and an area/molecule of $26 \pm 8 \AA$. The value of concentration is consistent to the values found by other authors, and as found in these studies, was slightly below in maximally dense mono-layer.,

The hydrophilic surfaces were prepared by performing the RCA 1 standard cleaning procedure at $75^{\circ} \mathrm{C}$, as described in our previous papers. ${ }^{4,6}$ The silicon oxide layers were characterised by ellipsometry and the average measured thickness was $14 \pm 4 \AA$.

Ellipsometry measures the changes in polarisation of an electromagnetic wave reflected from the experimental system, and has been thoroughly described in other works. ${ }^{18}$ To model the data, for hydrophilic surfaces we used a single-layer model for the silicon oxide layer prior to protein adsorption, and a two-layer

\footnotetext{
${ }^{\dagger}$ To whom correspondence should be addressed (E-mail: msferraz@ulb.ac.be).

${ }^{\dagger}$ Present address: Credit Swiss, London, UK
} 
model after the protein adsorption, while for hydrophobic surfaces a two-layer model for the $\mathrm{SiO}_{2} / \mathrm{OTS}$ grafted layer system and a three-layer model, after the protein adsorption, were used. For thin adsorbed layers it is not possible to extract both the density and thickness of the layer. However, a "total optical thickness" (which we call pseudo-thickness) of the layer can be measured accurately, and this can related to the concentration of the material within the layer through the equation of de Feijter ${ }^{19}$

$$
\Gamma=\frac{d\left(n-n_{0}\right)}{10(d n / d c)}
$$

where $d n / d c$ is the refractive index increment of the material in $\mathrm{mL} \mathrm{g}^{-1}, c$ is the concentration of the solution comprising the protein film in $\mathrm{g} \mathrm{ml}^{-1}, d$ is thickness in $\AA, n$ is the refractive index of the film, and $n_{0}$ is the refractive index of the aqueous medium in which the material in dissolved.

For the two-layer model, a second layer of constant refractive index of 1.4142 represented the adsorbed layer. This refractive index is equivalent to a volume fraction of protein of $\sim 33 \%$, the rest of the layer being water, as in our previous work. ${ }^{4}$ The only fitted parameter was the pseudo-thickness of the protein layer. The optical properties of the protein solutions satisfy the equation below:

$$
c=\frac{\left(n-n_{0}\right)}{(d n / d c)}
$$

The density of lysozyme was taken to be 1.405 $\mathrm{g} \mathrm{mL}^{-1}$, derived from the partial specific volume. ${ }^{20}$ From the pseudo-thickness of the adsorbed layer, the adsorbed amount can be calculated using the equation of de Feijter.

Spectroscopic ellipsometry measurements were recorded for every experiment prior the insertion of the protein solution in the cell, at the beginning to the adsorption time and at the end of the pre-determined adsorption time. The measurements were taken at an angle of 71 degrees, close to the Brewster angle of the system. The ellipsometer used was a Jobin-Yvon Unisel spectroscopic phase modulated ellipsometer. The light consists of a $75 \mathrm{~W}$ xenon lamp, producing bright white light from $2200 \mathrm{~nm}$ in the IR to $240 \mathrm{~nm}$ in the UV.

The cell used was thoroughly described in our previous work. ${ }^{4}$ The cellule was situated on a hot stage and the maximum heating rate is $5^{\circ} \mathrm{C} \mathrm{min}^{-1}$, and the cooling rate is $1^{\circ} \mathrm{C} \mathrm{min}^{-1}$.

The solutions were made of phosphate buffered saline at $\mathrm{pH}$ 7.0, ionic strength 0.1 . For the adsorption and the rinsing experiments, the pseudo-thicknesses were converted to adsorbed amounts using the equation of de Feijter. The conversion for lysozyme was effected by dividing the pseudo-thickness by $23.2 \AA$.

FT IR-ATR spectroscopy was used as a complementary technique to obtain some additional information. The instrument and the data analysis have been thoroughly described elsewhere., ${ }^{9,10}$ For these measurements, the silicon ATR crystals used for all experiments were treated in the same way that for the ellipsometreic experiments, including cleaning and surface oxidation using the RCA procedure, followed by hydrophobing with OTS.

\section{Heat-treatment of the Adsorbed Protein Layer}

We were interested in the effect of the heat treatment on adsorbed layers of lysozyme at surfaces. After the adsorption, we observed a pseudo-thickness of $56 \pm 5 \AA$ and $50 \pm 5 \AA$ on the hydrophilic and hydrophobic surfaces respectively. After the heating procedure, consisting of heating the system to $70^{\circ} \mathrm{C}$ for $1 \mathrm{~h}$, we did not observe significant changes from the initial values on the pseudo-thickness.

\section{Displacement Heated Lysozyme by $C_{12} E_{8}$}

The displacement of the heat-treated adsorbed lysozyme by $C_{12} E_{8}$ was investigated only on hydrophobic surfaces. This was because, as has been reported in our previous work, ${ }^{4}$ the surfactant has little influence at hydrophilic surfaces. The surfactant concentration was $1 \mathrm{mg} / \mathrm{mL}$. For the displacement of heated lysozyme by $C_{12} E_{8}$ the ellipsometry experiments give a pseudo-thickness, after the heating treatment at $70^{\circ} \mathrm{C}$ for $1 \mathrm{~h}$, of $44 \pm 3 \AA$. After the introduction of the surfactant, we observed an increase in pseudothickness of around $20 \AA$. Heating was always performed in an ambient medium of pure buffer solution. After a displacement time of $1000 \mathrm{~min}$, the pseudothickness remained at least $14 \AA$ above the value at the end of heat-treatment, as shown in Figure 1 (right).

The increase of at least $14 \AA$ in pseudo-thickness

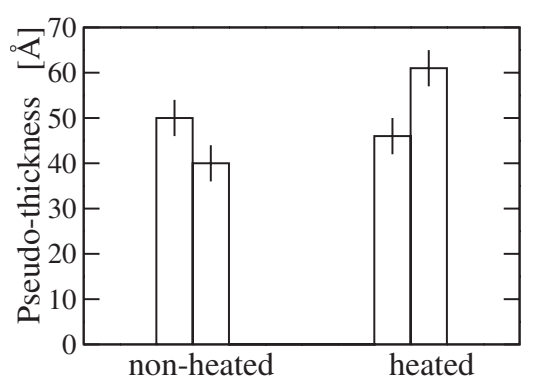

Figure 1. Pseudo-thickness for non-heated (on the left part of the figure) and heated (on the right part of the figure) lysozyme layers during the surfactant displacement experiments. On both the left and right sides, the pseudo-thickness prior to the insertion of the surfactant (left) and after a surfactant exposure time of 1000 min (right) are shown. 
persisted throughout the displacement procedure for the heated layer, in contrast to the non-heated experiments. This experiment was indeed performed as similar to the heating one and the results are shown in Figure 1 on the left where instead we observed a reduction in final displacement pseudo-thickness, as a percentage of the final rinsed measurement, of around $15 \%$. This value is much smaller then those previously reported for beta-lactoglobulin. ${ }^{4}$ This suggests that lysozyme binds more strongly to the hydrophobic surface than beta-lactoglobulin and is less easily displaced by surfactant.

Our results also indicate that upon adding the surfactant solution, a surfactant layer quickly and permanently adsorbed on top of the heat-treated layer of lysozyme with minimal displacement, opposite to the observation of the non-heated case.

\section{FTIR-ATR Characterisation of Heated Adsorbed Lysozyme}

The FT IR-ATR technique was used to investigate the heat-induced changes of the adsorbed lysozyme on the hydrophobic surface, and their displacement by $C_{12} E_{8}$. Our FTIR-ATR experiments were performed under the same conditions as the ellipsometric experiments. We observed that the heating of adsorbed lysozyme at the hydrophobic surface almost doubled the amide I peak area (Figure 2). There are also large changes in peak shape. These changes in peak area and shape may be caused by changes within the adsorbed layer connected to surface gelation rather than by additional protein adsorbing during the heating, as showed by the corresponding ellipsometry experiments indicating that the adsorbed amount of lysozyme did not significantly change due to heat treatment. Ball and Jones ${ }^{9}$ have attributed the pronounced shoulder at $1610-16201 / \mathrm{cm}$ to the formation of beta-sheet secondary structure upon gelation, although that work took place on a hydrophilic surface. It should be noted that there might be differences in response of adsorbed lysozyme to heat-treatment on

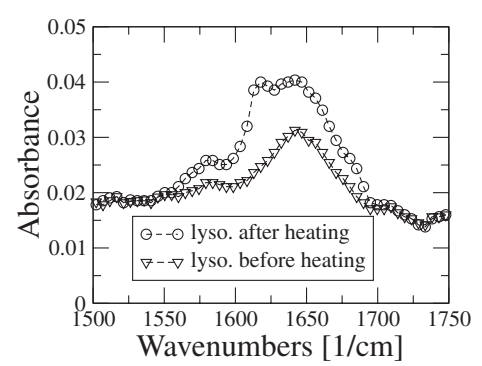

Figure 2. The figure shows FT IR-ATR spectra of an adsorbed layer of lysozyme before and after heat-treatment at $70^{\circ} \mathrm{C}$ for $1 \mathrm{~h}$. The large increase in amide I peak area, together with an additional shoulder, are apparent (in the legend lyso. stays for lysozyme). hydrophobic surfaces. For example, the temperatures of onset of gelation or unfolding may be lower on the hydrophobic surface, due to the surface promoting greater conformational changes in adsorbed molecules leading to lower thermal stability. For comparison, the amide I peak for $\beta$-lactoglobulin on a hydrophobic surface was different from lysozyme showing much stronger absorbance around $1610-16201 / \mathrm{cm}$, the range associated with $\beta$-sheet formation during gelation of lysozyme. The nature structure of for $\beta$-lactoglobulin has in fact $43 \% \beta$-sheet structure, compared with only $16 \%$ for lysozyme. ${ }^{20}$ These insights would form very interesting work for the future.

The displacement by $C_{12} E_{8}$ of an adsorbed, heated lysozyme layer was also characterised by ATR experiments. In the absence of the heating effect described earlier, the Amide I peak area measures concentration of protein alone, and is not affected by surfactant. In contrast, the optical technique of ellipsometry cannot distinguish between surfactant and protein. The FTIR-ATR results showed a small reduction of $10 \pm 4 \%$ of the amide I peak area during displacement. This shows that $90 \pm 4 \%$ of the heat-treated lysozyme was not removed by surfactant, and supports the observation made from ellipsometry that most of the increase in pseudo-thickness is due to surfactant adsorbing in addition to the existing protein layer.

These results, together with those for ellipsometry, shows when heating the lysozyme layer to $70^{\circ} \mathrm{C}$, the protein layer showed more resistance to displacement indicating that protein is more strongly adsorbed. This stronger binding to the surface is probably caused by conformation changes in adsorbed molecules due to further unfolding and heat-set gelation at the surface. ${ }^{9}$ Our work adds to the accumulating body of evidence supporting the importance of such structural changes to the interfacial behaviour of some of the most fascinating and important biological polymers, the globular proteins.

\section{REFERENCES}

1. a) J. L. Brash and T. A. Horbett, Eds., "Proteins at Interfaces. Physicochemical and Biochemical Studies," Vol. 343, ACS, Washington, D.C., 1987.

b) T. A. Horbett and J. L. Brash, Eds., "Proteins at Interfaces II. Fundamentals and Applications," Vol. 602, ACS, Washington, D.C., 1995.

2. W. Norde, Proteins at solid interfaces, in: A. Baszkin and W. Norde, Eds., "Physical Chemistry of Biological Interfaces," Marcel Dekker, New York, 2000.

3. J. J. Gray, Curr. Opin. Struct. Biol., 14, 110 (2004).

4. R. J. Marsh, R. A. L. Jones, and M. Sferrazza, Colloids. Surf., B, 23, 31 (2002).

5. a) M. Malmsten, Colloids. Surf., B, 3, 371 (1995). b) M. Malmsten, Colloids. Surf., B, 3, 297 (1995). 
6. R. J. Marsh, R. A. L. Jones, and M. Sferrazza, J. Colloid Interface Sci., 218, 347 (1999).

7. G. Fragneto et al., Langmuir, 12, 477 (1996).

8. A. R. Rennie et al., Science, 367, 657 (1995).

9. A. Ball and R. A. L. Jones, Langmuir, 11, 3342 (1995).

10. R. J. Green, I. Hopkinson, and R. A. L. Jones, Langmuir, 15, 5102 (1999).

11. J. S. Sharp, J. A. Forrest, and R. A. L. Jones, Biochemistry, 41, 15810 (2002).

12. C. E. Giacomelli and W. Norde, J. Colloid Interface Sci., 233, 234 (2001).

13. P. B. Welzel, Thermochim. Acta, 382, 175 (2002).
14. P. Billsten et al., Langmuir, 15, 6395 (1999).

15. T. Imoto et al., "The Enzyme,” P. D. Boyer, Ed., Academic Press, New York, 1972, p 665.

16. F. Tiberg et al., Langmuir, 10, 2294 (1994).

17. D. R. Lide and H. P. R. Frederikse, "CRC Handbook of Chemistry and Physics," CRC Press, London, 1994.

18. D. Styrkas et al., in: R. W. Richards and S. K. Peace, Eds., "Polymer Surfaces and Interfaces III," Wiley \& Sons, New York, 1999.

19. J. A. de Feijter et al., Biopolymers, 17, 1759 (1978).

20. M. C. Wahlgren et al., Colloids Surf., A, 70, 139 (1993). 